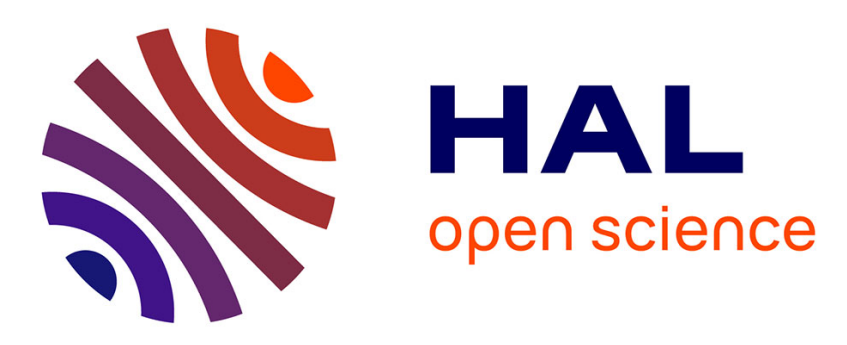

\title{
Energy Efficient Massive MIMO Point-to-Point Communications with Physical Layer Security: BPSK vs QPSK Decomposition
}

\author{
David Borges, Paulo Montezuma, Rui Dinis, Pedro Viegas
}

\section{To cite this version:}

David Borges, Paulo Montezuma, Rui Dinis, Pedro Viegas. Energy Efficient Massive MIMO Point-toPoint Communications with Physical Layer Security: BPSK vs QPSK Decomposition. 10th Doctoral Conference on Computing, Electrical and Industrial Systems (DoCEIS), May 2019, Costa de Caparica, Portugal. pp.283-295, 10.1007/978-3-030-17771-3_25 . hal-02295261

\section{HAL Id: hal-02295261 \\ https://hal.inria.fr/hal-02295261}

Submitted on 24 Sep 2019

HAL is a multi-disciplinary open access archive for the deposit and dissemination of scientific research documents, whether they are published or not. The documents may come from teaching and research institutions in France or abroad, or from public or private research centers.
L'archive ouverte pluridisciplinaire HAL, est destinée au dépôt et à la diffusion de documents scientifiques de niveau recherche, publiés ou non, émanant des établissements d'enseignement et de recherche français ou étrangers, des laboratoires publics ou privés.

\section{(c)(1)}

Distributed under a Creative Commons Attribution| 4.0 International License 


\title{
Energy Efficient Massive MIMO Point-to-Point Communications with Physical Layer Security: BPSK vs QPSK Decomposition *
}

\author{
David Borges $^{1}$, Paulo Montezuma ${ }^{1,2}$, Rui Dinis ${ }^{1,2}$, Pedro Viegas ${ }^{2}$ \\ ${ }^{1}$ DEE, FCT Universidade Nova de Lisboa, Portugal \\ ${ }^{2}$ IT, Instituto de Telecomunicações, Av. Rovisco Pais, Lisboa, Portugal
}

\begin{abstract}
Massive multiple-input multiple-output systems (mMIMO) are the most prevalent candidates for the next generation of wireless communication. Yet even with mMIMO systems the joint optimization of spectral and energy efficiencies can be only attained by combining high order signal constellations and efficient power amplification. In order to push this limitation, the transmitter can spread the information into several amplification branches, which are the result of the decomposition of multilevel constellation symbols into quasi constant envelope signals. Nevertheless, the high number of antennas involved in this type of communication leads to an increase of the channel matrix's size and therefore the complexity of the equalization process can create drawbacks for the power consumption and latency. In this paper we will study the combination of a multi-layer transmitter with a low complexity receivers based on an iterative block decision feedback equalizer (IB-DFE). These receivers avoid the matrix inversion operation in the equalizer the feed-forward by replacing it with an equal gain combiner (EGC) or a maximum ratio combiner (MRC) module. Results show that can be used without penalties on performance provided that the number of antennas involved is high.
\end{abstract}

Keywords: massive MIMO systems, efficient power amplification, constellation shaping, low complexity detection.

\section{Introduction}

Massive multiple-input multiple-output (mMIMO) systems allow many improvements in the communication performance bounds [1]. Additionally, to maximize spectral efficiency, high order multilevel constellations can be employed. However, high order multilevel constellations have high peak-to-average power ratio (PAPR) which restricts the efficiency of the amplification [2]. In order to improve the power amplification efficiency, the symbols with high envelope fluctuations can be decomposed into low order modulation symbols, which benefit of low envelope

\footnotetext{
* This work was supported in part by FCT - Portuguese Foundation for Science and Technology through the PhD scholarship SFRH/BD/131093/2017, IT UID/EEA/50008/2013 (plurianual founding and project GLANCES), EnAcoMIMOCo EXPL/EEI-TEL/2408/2013 and UID/EEA/50008/2013 - MM5G.
} 
fluctuations [3]. As example, we can decompose one 16 Quadrature Amplitude Modulation (QAM) symbol into four Binary Phase Shift Keying (BPSK) symbols or two Quadrature Phase Shift Keying (QPSK) symbols. By doing this, each component can be amplified separately without distortion by NonLinear (NL) power amplifiers, since the PAPR of a BPSK or a QPSK is much lower than the PAPR of the original constellation. Using not oversized amplifiers and working in their optimal operation zone, energy efficiency of each amplifier can be highly improved. In this architecture, each power amplifier is directly connected to a single antenna. This means that each antenna will transmit a single component from the decomposed higher order symbol, i.e. a BPSK or QPSK symbol. At the channel level, the original symbol can be obtained through the combination of all the components. In other words, at the receiver side, each antenna will receive the signal from all transmitting antennas which will add up to form the original high order constellation symbol. Moreover, a multi-layer system could be created using this approach. The first layer is composed by the multi-branch amplification structure, the second layer is composed by the antenna array connected to each amplifier's output and the third layer associated to spatial multiplexing is composed by several layers 1 and 2 in parallel.

However, the high number of antennas employed in the multi layered transmitter and receiver communication process will imply an increasing size of the channel matrix, which means a higher complexity in the equalization step due to matrix inversion operation. In order to overcome this problem, a low complexity receiver, combining an Iterative Block Decision Feedback Equalization (IB-DFE) and a Maximum Ratio Combiner (MRC) or an Equal Gain Combiner (EGC) is implemented for systems based on these multi layered transmitters and analyzed in this paper. These simplified receivers avoid the usual matrix inversion inherent to mMIMO conventional detection scheme, but as we shall see the conditions for such approach must be fulfilled to avoid any impact on system performance.

The rest of this paper is organized as follows: Section 2 characterizes the system's architecture; Section 3 contains the description of the system; Simulation results and performance analysis are presented in section 4; Section 5 resumes the paper.

\section{System Architecture and Characterization}

It was already shown that hig order constellations can be decomposed in polar components with the constellation symbols expressed as a function of the corresponding bits [4]. Let $\mathcal{S}=\left\{s_{0}, s_{1}, \ldots, s_{(\mathrm{N}-1)}\right\}$, a constellation with $M$ points (i.e., $\# \mathcal{S}=M$ ), where $s_{n} \in \mathbb{C}$. To each constellation point $s_{n}$ we associate a set of $\mu$ $=\log _{2}(M)$ bits in polar form $\mathcal{B}=\left\{b_{n}^{0}, b_{n}^{1}, \ldots, b_{n}^{(\mu-1)}\right\}$, with $b_{n}^{(i)}= \pm 1=2 \beta_{n}^{(i)}-$ 1 and $\beta_{n}^{(i)}=0$ or 1 . The set of $\mu$ bits can be decomposed in $M=2^{\mu}$ different subsets $\mathcal{B}_{m}, m=0,1, \ldots, M-1$.

Having $M$ constellation points in $\mathcal{S}$ and $M$ different subsets of $\mathcal{B}, \mathcal{B}_{0}, \mathcal{B}_{1}, \ldots$, $\mathcal{B}_{M-1}$, we can write 


$$
\mathrm{s}_{\mathrm{n}}=\sum_{\mathrm{m}=0}^{\mathrm{M}-1} \mathrm{~g}_{\mathrm{m}} \prod_{\mathrm{b}_{\mathrm{n}}^{(\mathrm{i})} \in \mathcal{B}_{\mathrm{m}}} \mathrm{b}_{\mathrm{n}}^{(\mathrm{i})}, \mathrm{n}=0,1, \ldots, \mathrm{M}-1,
$$

which corresponds to a system of $M$ equations (one for each $s_{n}$ and $M$ unknown variables $\left.g_{m}\right)$. Moreover, by doing $m=\left(\gamma_{(\mu-1, m)}, \gamma_{(\mu-2, m)}, \ldots, \gamma_{(1, m)}, \gamma_{(0, m)}\right)$ we can associate $m$ to its corresponding binary representation with $\mu$ bits, which turns possible to define $\mathcal{B}_{m}$ as the set of bits where the bit $b_{n}^{(i)}$ is included if and only $\gamma_{(i, m)}$ is 1 . Based on that we may write

$$
s_{n}=\sum_{m=0}^{M-1} g_{m} \prod_{i=0}^{\mu-1}\left(b_{n}^{(i)}\right)^{\gamma_{(i, m)}} .
$$

The transmitter layered structure of Fig. 1 takes advantage of this decomposition in polar components, and uses a mMIMO scheme with $N_{v} \times N_{m} \times N_{b}$ antenna elements, arranged in $N_{v}$ sets of $N_{m} \times N_{b}$ antennas. Conventional beamforming schemes could be implemented by a layer 2 with $N_{b}$ antenna elements connected to each one of $N_{m}$ amplification branches. For spatial multiplexing a layer 3 with a set of $N_{v} \times N_{m}$ antennas is needed, where the $N_{m}$ antennas of layer 1 are associated to the signal components of the constellation symbol and $N_{v}$ sets of $N_{m}$ antennas are used to transmit simultaneously $N_{v}$ different constellation symbols (an example of a transmitter based on a layer 1 and layer 3 combination can be seen in Fig. 2).

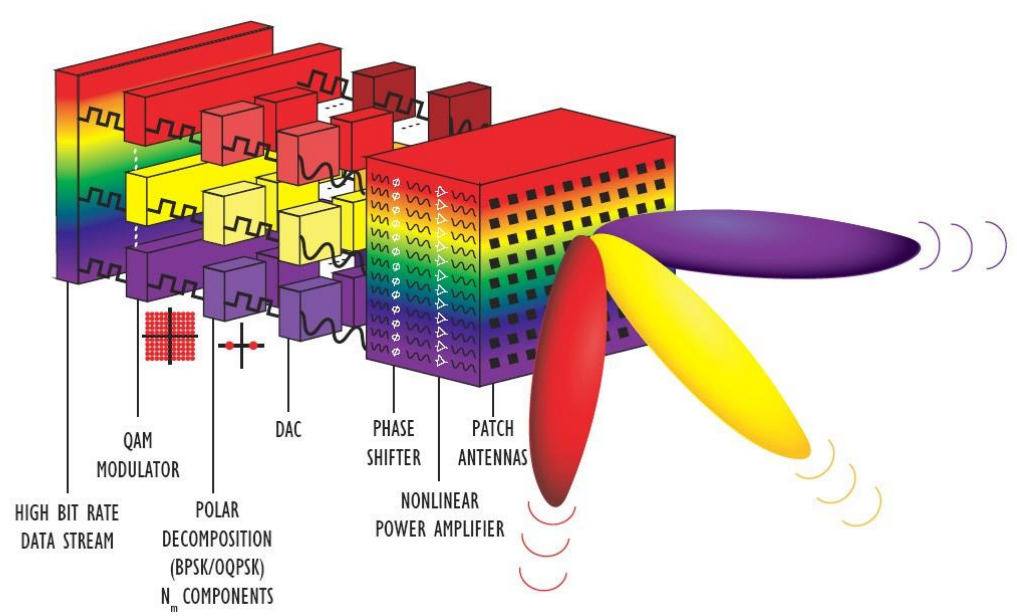

Fig.1. Transmitter layered structure.

It follows that power efficiency comes improved due the lower PAPR of the component signals and the possible use of NL amplifiers in layer 1 [3,5]. Another important aspect lies on uncorrelated signals associated to each RF chain, which 
means an omnidirectional radiation pattern for each set of $N_{m}$ antennas. But it is important to mention that each set of $N_{m}$ antennas implements directivity at information level, since the constellation points of the transmitted signal maintain their positions at the desired direction $\Theta$ but are scrambled for other directions.

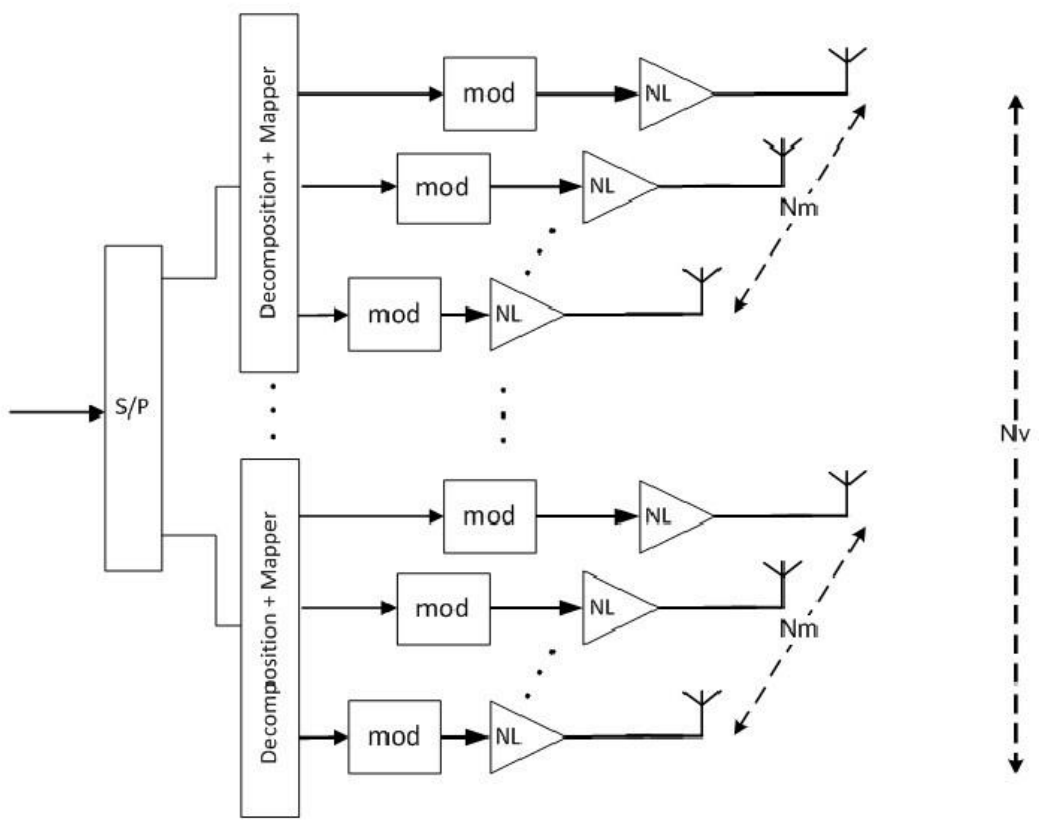

Fig. 2. Layered transmitter structure with layer 1 and layer 3.

In Fig. 3 it can be seen that the transmitted components will arrive to the receiver with different delays, related with the spacing $d$ between antennas and the desired transmission angle $\Theta$. However, these delays are compensated by introducing phase shifts between antennas related to the spacing between the amplification branches in the transmitter. This means that in direction $\Theta$ the receiver sees a symbol constellation without any distortion due to phase rotations of signal components. In other directions, phase shifts between antennas do not compensate the phase shifts due the different propagation delays and the received symbol suffers distortions since it results from the combination of signal components with the wrong phases. Obviously antenna spacing can be adjusted to assure the desired configuration of information directivity for a specific direction. Therefore, any user can demodulate the received signal if is in the desired direction while other users in other directions receive a scrambled constellation. Hence, to decode the information, each $j$ user needs to be in the right direction $\Theta_{j}$. 


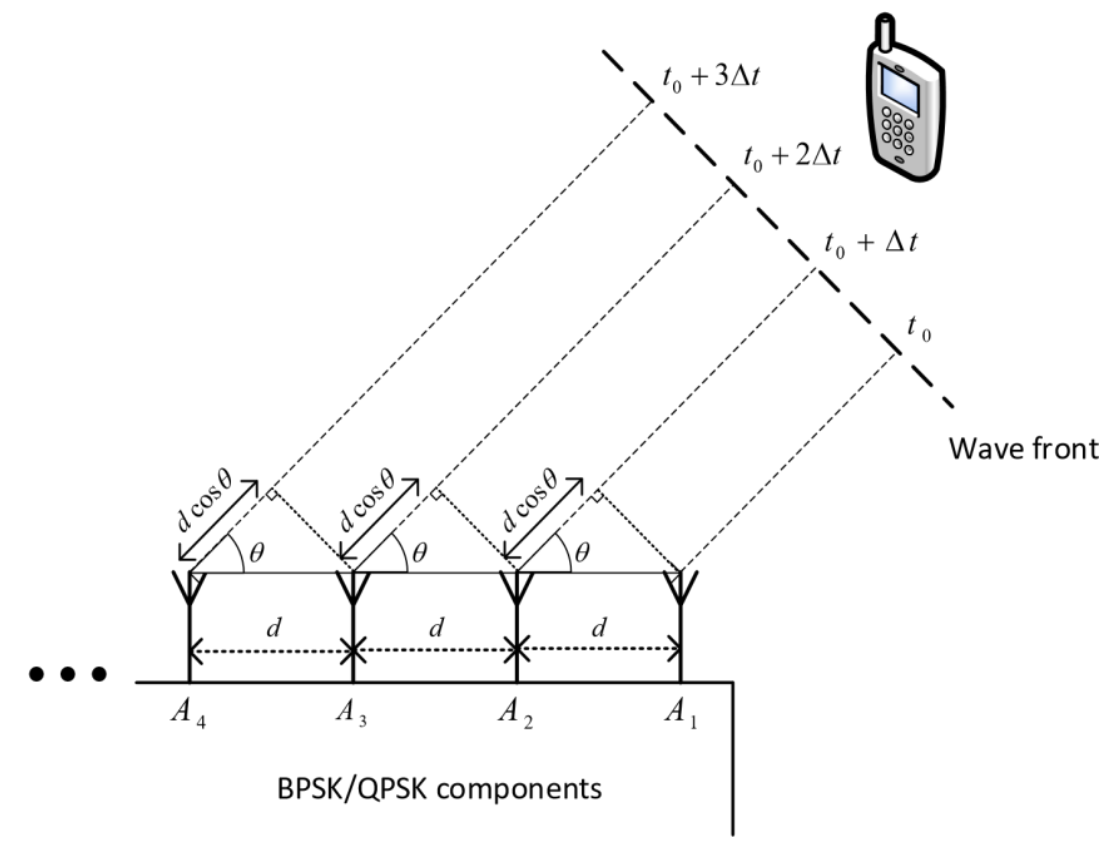

Fig. 3. Constellation rotation due to delay.

\section{System Description}

Consider now the MIMO scenario shown in fig. 4 characterized by a point-to-point communication link between a transmitter with $T=N_{v} \times N_{m}$ antennas and a receiver with $R \geq T$ receive antennas. The transmitter's configuration has the layered structure shown in fig. 2, but were Layer 3 is composed by a set of $N_{v}$ transmitters each one composed by $N_{m}$ amplification branches in parallel (for a 16-quadrature amplitude modulation (16-QAM) decomposition into QPSK or BPSK components we have $N_{m}=2$ and $N_{m}=4$, respectively). This means a third layer with $T=$ $N_{v} \times N_{m}$ antennas that transmits simultaneously $N_{v}$ symbol constellations (more exactly the components of $N_{v}$ symbols from a 16-QAM constellation). The transmitter configuration is known and the receiver is assumed to be in the right direction $\Theta$ (in which the constellation is optimized).

Coupling effects among antennas are avoided by a horizontal spacing of $\lambda / 2$ between antennas of layer 1 and a vertical spacing of $\lambda$ between each set of $N_{m}$ antennas. It is assumed that all antennas at transmitter are assigned to an authorized receiver with $R$ antennas.

It is assumed that we have frequency slective channels linking transmit and receive antennas and a SC-FDE block transmission technique. To compensate the channel's 
frequency selectivity it can be adopted an IB-DFE receiver with the structure shown in fig. $3[6,7]$.

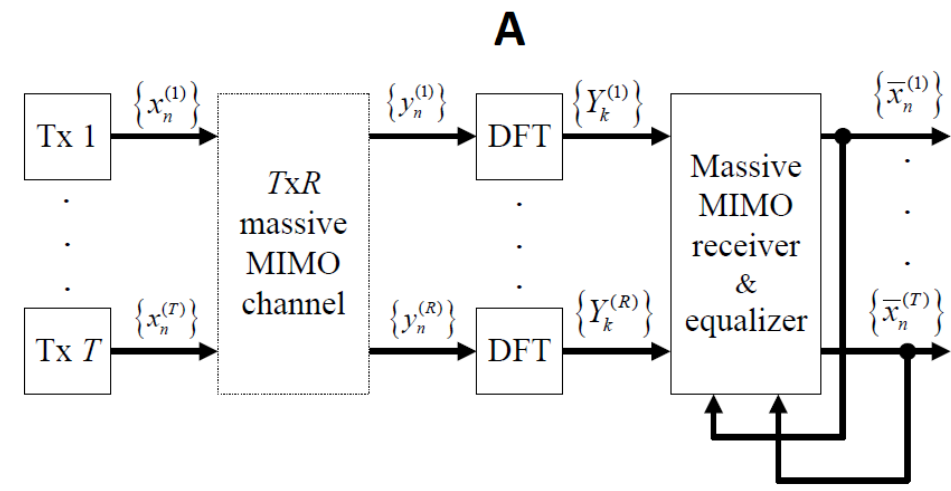

B

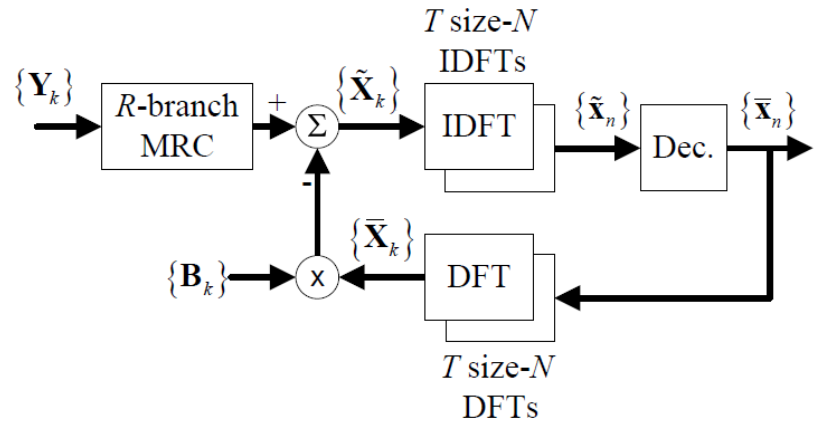

Fig. 4. Massive MIMO system architecture for SC-FDE schemes (A) and detail of the low complexity massive MIMO receiver and equalization (B).

At transmitter the $t^{\text {th }}$ antenna sends the block of $N$ data symbols $\left\{x_{n}^{(t)} ; n=\right.$ $0,1, \ldots, N-1\}$ being $\left\{y_{n}^{(r)} ; k=0,1, \ldots, N-1\right\}$ the received block at the $r$ th receiver's antenna. As usual, a cyclic prefix with duration higher than the overall channel delay is appended to each transmitted block and removed at the receiver. Under these conditions, the corresponding frequency-domain received block $\left\{Y_{k}^{(r)} ; k=0,1, \ldots, N-1\right\}$ is given by

$$
\mathbf{Y}_{k}=\left[\begin{array}{llll}
Y_{k}^{(1)} & \ldots & Y_{k}^{(R)}
\end{array}\right]^{T}=\mathbf{H}_{k} \mathbf{X}_{k}+\mathbf{N}_{k},
$$

where $\mathbf{H}_{k}$ denotes the $R \times T$ channel matrix for the $k$ th frequency, with $(r, t)$ th element $H_{k}^{(r, t)}, \mathbf{X}_{k}=\left[\begin{array}{lll}X_{k}^{(1)} & \ldots & X_{k}^{(T)}\end{array}\right]^{T}$ and $\mathbf{N}_{k}$ represents the channel noise. 
In an iterative Minimum Mean Squared Error (MMSE) receiver the data symbols in each iteration are obtained through the inverse discrete Fourier transform (IDFT) of the block $\left\{\tilde{X}_{k}^{(t)} ; k=0,1, \ldots, N-1\right\}$, and are given by

$$
\widetilde{\mathbf{X}}_{k}=\left[\tilde{X}_{k}^{1} \ldots \tilde{X}_{k}^{(R)}\right]^{T}=\mathbf{F}_{k} \mathbf{Y}_{k}-\mathbf{B}_{k} \overline{\mathbf{X}}_{k}
$$

(see details in [8], e.g.). Interference cancelation is done using $\overline{\mathbf{X}}_{k}=\left[\bar{X}_{0} \ldots \bar{X}_{N-1}\right]$, with $\bar{X}_{k}$ denoting the frequency-domain average values conditioned to the FDE output for the previous iteration, which can be computed as described in $[9,8]$. Coefficients $F_{k}, B_{k}$ and the correlation coefficient $\rho$ can be computed as described in $[4,7,8]$. In first iteration no information is available about the transmitted symbols and $\overline{\mathbf{X}}_{k}=\mathbf{0}$, which means that this receiver is equivalent to the linear frequency-domain MMSE receiver. Subsequent iterations use the average values conditioned to the receiver's output from previous iteration to remove the residual intersymbol interference (ISI).

Due to matrix inversions the IB-DFE receiver has a computational complexity of $O\left(N_{R}{ }^{3}\right)$. To avoid this problem other two low-complexity iterative frequency-domain receivers, denoted as maximum ratio detection (MRD) and equal gain detection (EGD) are also considered [10]. Similarly to [10] the ratios $R / T$ between receiving and transmitting antennas considered here are at least equal or higher than 4 .

As mentioned in [10], the MRD receiver (see Fig. 3 B) is characterized by

$$
\widetilde{\mathbf{X}}_{k}=\Psi \mathbf{H}_{k}^{H} \mathbf{Y}_{k}-\mathbf{B}_{k} \overline{\mathbf{X}}_{k}
$$

and

$$
\mathbf{B}_{k}=\boldsymbol{\Psi} \mathbf{H}_{k}^{H} \mathbf{H}_{k}-\mathbf{I},
$$

where $\boldsymbol{\Psi}$ denotes a diagonal matrix in which the $(t, t)$ th element is given by $\left(\sum_{k=0}^{N-1} \sum_{r=1}^{R}\left|H_{k}^{(r, t)}\right|^{2}\right)^{-1}$, takes advantage of the fact that

$$
\mathbf{H}_{k}^{H} \mathbf{H}_{k} \approx R \mathbf{I},
$$

which is accurate when $R>>1$ and the correlation between different channels have small correlation (i.e., different transmit and receive antennas). On the other hand, the EGD characterized by

$$
\mathbf{B}_{k}=\boldsymbol{\Psi} \mathbf{A}_{k}^{H} \mathbf{H}_{k}-\mathbf{I} \text {, }
$$

where $\boldsymbol{\Psi}$ denotes a diagonal matrix whose $(t, t)$ th element is given by $\left(\sum_{k=0}^{N-1} \sum_{r=1}^{R}\left|H_{k}^{(r, t)}\right|\right)^{-1}$, takes advantage of the fact that for mMIMO systems with $R \gg 1$ and small correlation between the channels associated to different transmit and receive antennas, the elements outside the main diagonal of

$$
\mathbf{A}_{k}^{H} \mathbf{H}_{k}
$$


are much lower than the ones at its diagonal, where $\left(i, i^{\prime}\right)$ th element of the matrix $\mathbf{A}$ is $[\mathbf{A}]_{i, l}=\exp \left(\operatorname{jarg}\left([\mathbf{A}]_{i, l}\right)\right)$.

\section{Results}

Bit error rate (BER) results are obtained through Monte Carlo experiments (at least 150 error events are considered for each value of BER). A 16-QAM constellation is adopted in Layer 1, where the decomposition applied can be based on QPSK or BPSK components. This means that in first case we have $N_{m}=2$ branches with QPSK components and in second case we have $N_{m}=4$ BPSK components. In both cases the transmitted symbols $s_{n}$ are selected with equal probability from the original constellation. It is assumed linear power amplification at the transmitter and perfect time and frequency synchronization conditions at the receiver. The results are expressed as function of $\frac{E_{b}}{N_{0}}$, where $N_{0} / 2$ denotes the noise variance and $E_{b}$ represents the energy of the transmitted bits. The number of users is always settled at $T=5$. For EGD, MRD and IB-DFE receivers the number of iterations is 4 (previous study and showed than there is no advantage to consider higher number of iterations, since there are no significant improvements in performance) [10].

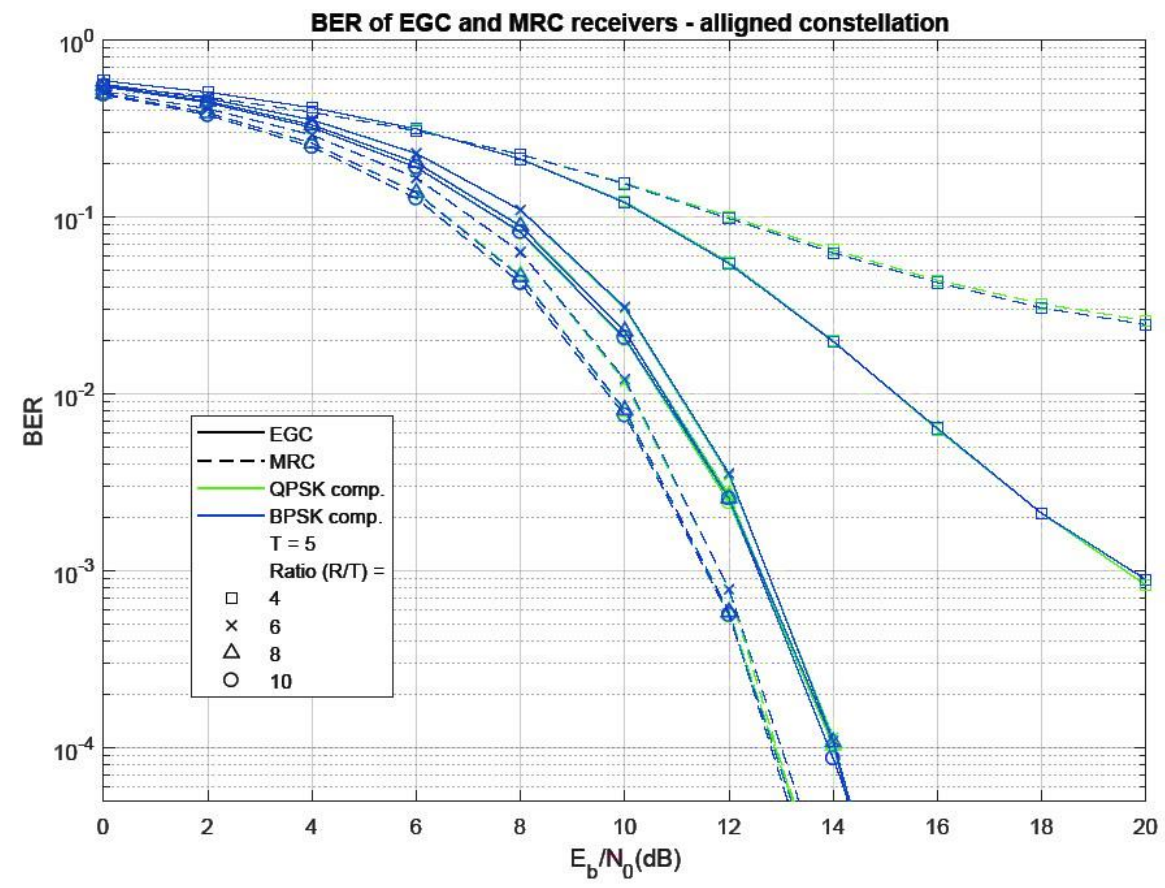

Fig. 5. BER performance for MRD and EGD with $\Delta \Theta=0^{\circ}$ and variant $R / T$. 


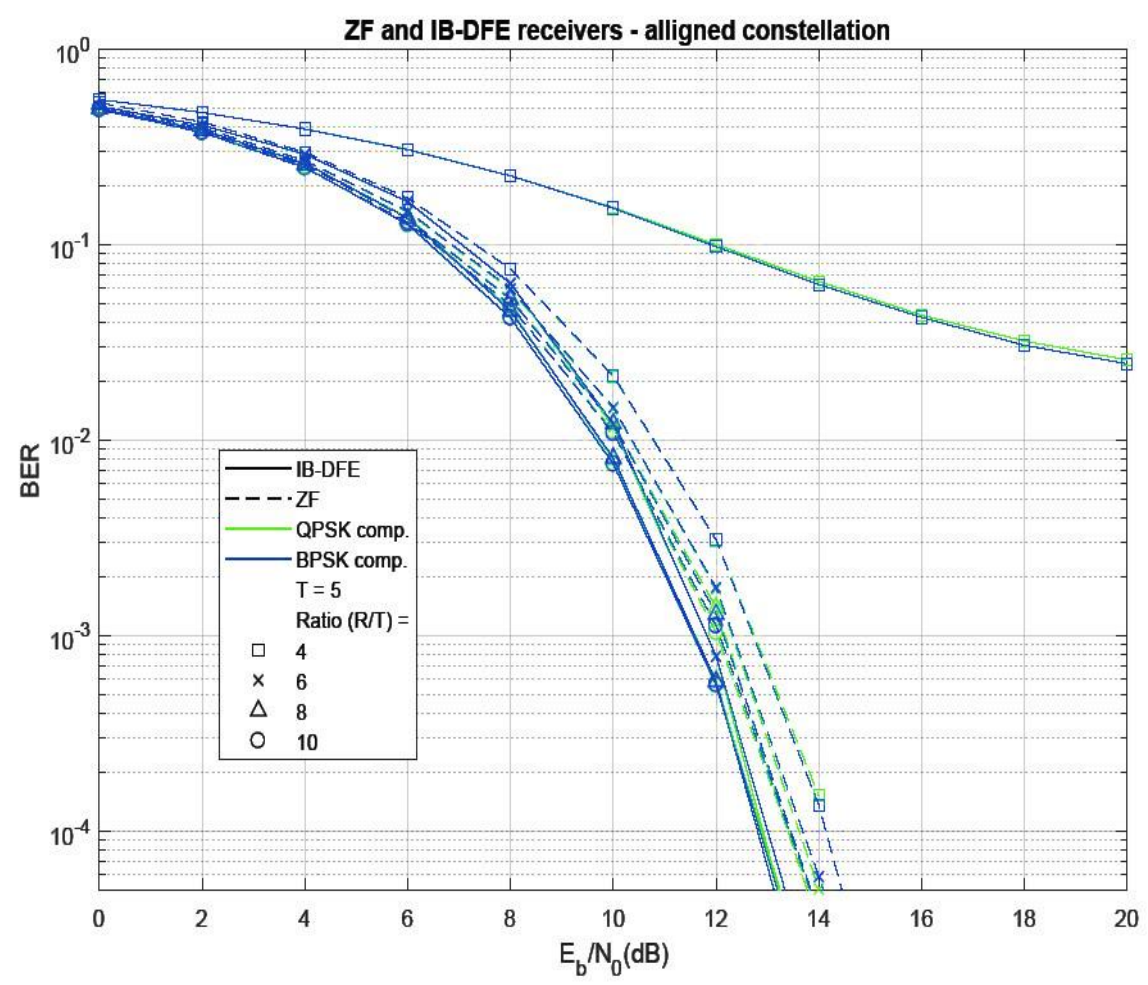

Fig. 6. BER performance for IB-DFE and ZF with $\Delta \Theta=0^{\circ}$ and variant $R / T$.

Results shown in Figs. 5 and 6 refer a layer 1 with both $N_{m}=2$ and $N_{m}=4$ and angular separation to the direction $\Theta$ of $\Delta \Theta=0^{\circ}$ (i. e. the user is placed in the correct direction where the components add in phase). Values of $R / T$ between 4 and 10 are also adopted. From the comparison of the results, it is clear that when the ratio $R / T$ grows, the performance improvement in $\mathrm{ZF}$ receiver can be considered as negligible. However for the other receivers only values of $R / T$ higher than 6 turn performance improvements to be considered as negligible (this means that the validity conditions for the approach done to avoid the matrix inversion stands for $R / T$ equal or higher than 6). As expected ZF and EGD perform worst that IB-DFE and MRD receivers, with the two later ones showing similar performances. Another important conclusion is the fact there is no performance disparity between both cases adopted for layer 1, since performances for the decomposition into BPSK and QPSK components are the same. It is important to mention, that the results for $\Delta \Theta=0^{\circ}$ are the same obtained by a conventional spatial multiplexing scheme, which means that combination of layer 1 and layer 3 can be employed without compromising system's performance.

To analyze the impact of errors in the alignment of the users with the direction $\Theta$, we consider a fixed ratio of $R / T=6$ and two values of $\Delta \Theta\left(0^{\circ}\right.$ and $\left.5^{\circ}\right)$. The results are presented in Figs. 7, 8 and 9 for different receivers compared with ZF. From the comparison of figures, it becomes clear that when the ratio $R / T$ grows, the gap 
between the MRD and the EGD receiver vanishes (as we can see in Figs. 7 and 8 performance curves of receivers overlap which is not the case of Fig. 5). It is also clear that despite the performance improvement achieved by the higher value of $R / T$, the performance impact of constellation shaping due to the decomposition of the constellation into into BPSK components is higher than QPSK case. Indeed, when at layer 1 , it is adopted a decomposition in BPSK components the data link becomes compromised for the ZF receiver, due to a BER of 0.5. Even the other receivers only have reasonable values of BERs for the decomposition into QPSK components. It is also observed that the IB-DFE exhibits more robustness to the constellation shaping, even for the angular error of $\Delta \Theta=5^{\circ}$. On the other hand, both MRD and EGD are severely affected by the angular error. Also, as expected the IB-DFE and the MRD receivers perform better than the EGD one. From the results of Figs. 7, 8 and 9 it is clear that both IB-DFE and MRD receivers have similar performances. Also, as expected, these two receivers perform better than the EGD one. On the other hand, better security is assured. This can be seen in the results regarding the ZF and EGD receivers where it is obvious the higher impact on system performance of any angular error for $N_{m}=4$. For $\Delta \Theta=5^{\circ}$ the BER near 0.5 means that users can't decode any useful information.

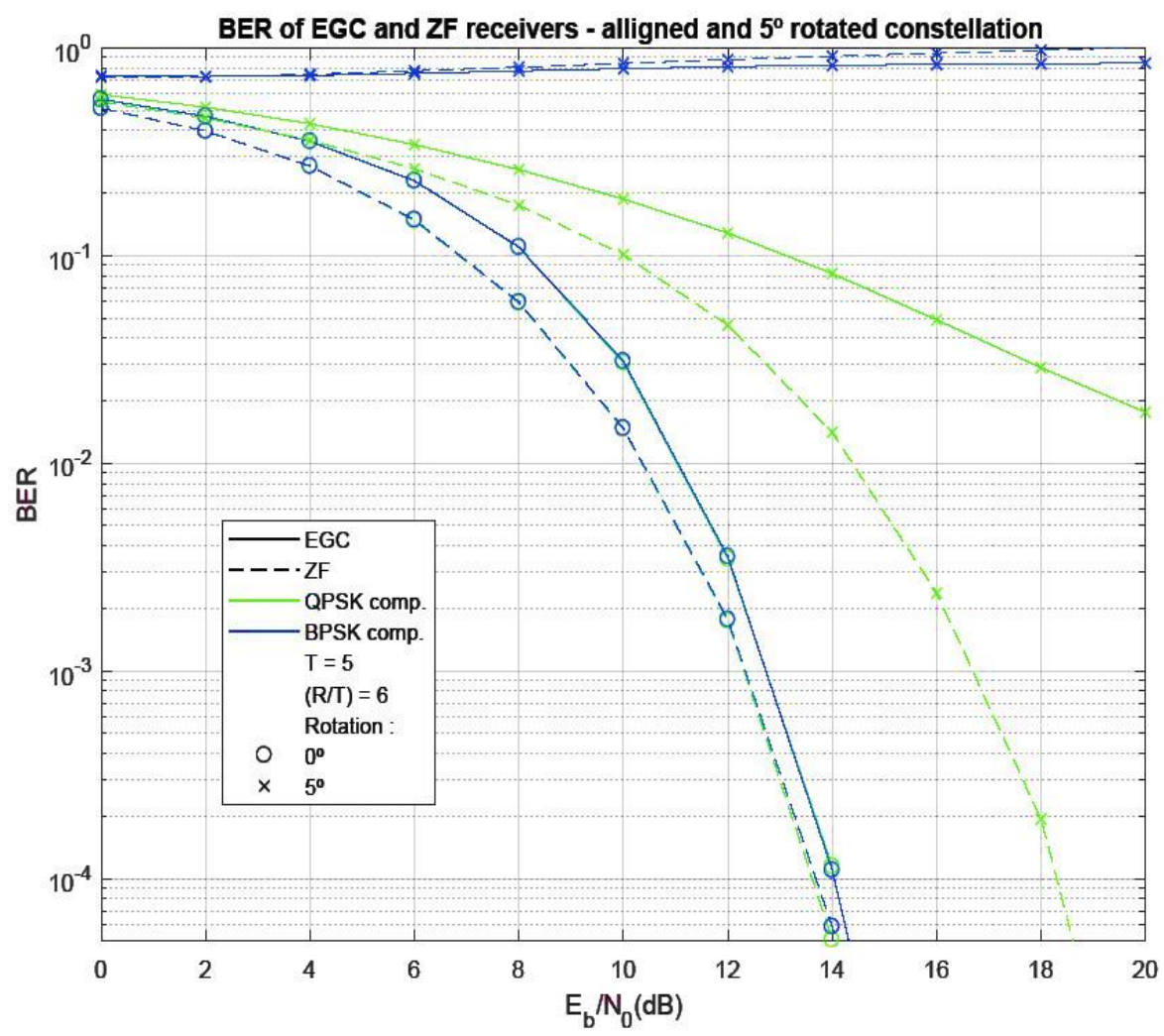

Fig. 7. BER performance comparing ZF and EGD with diferrent $\Delta \Theta$. 


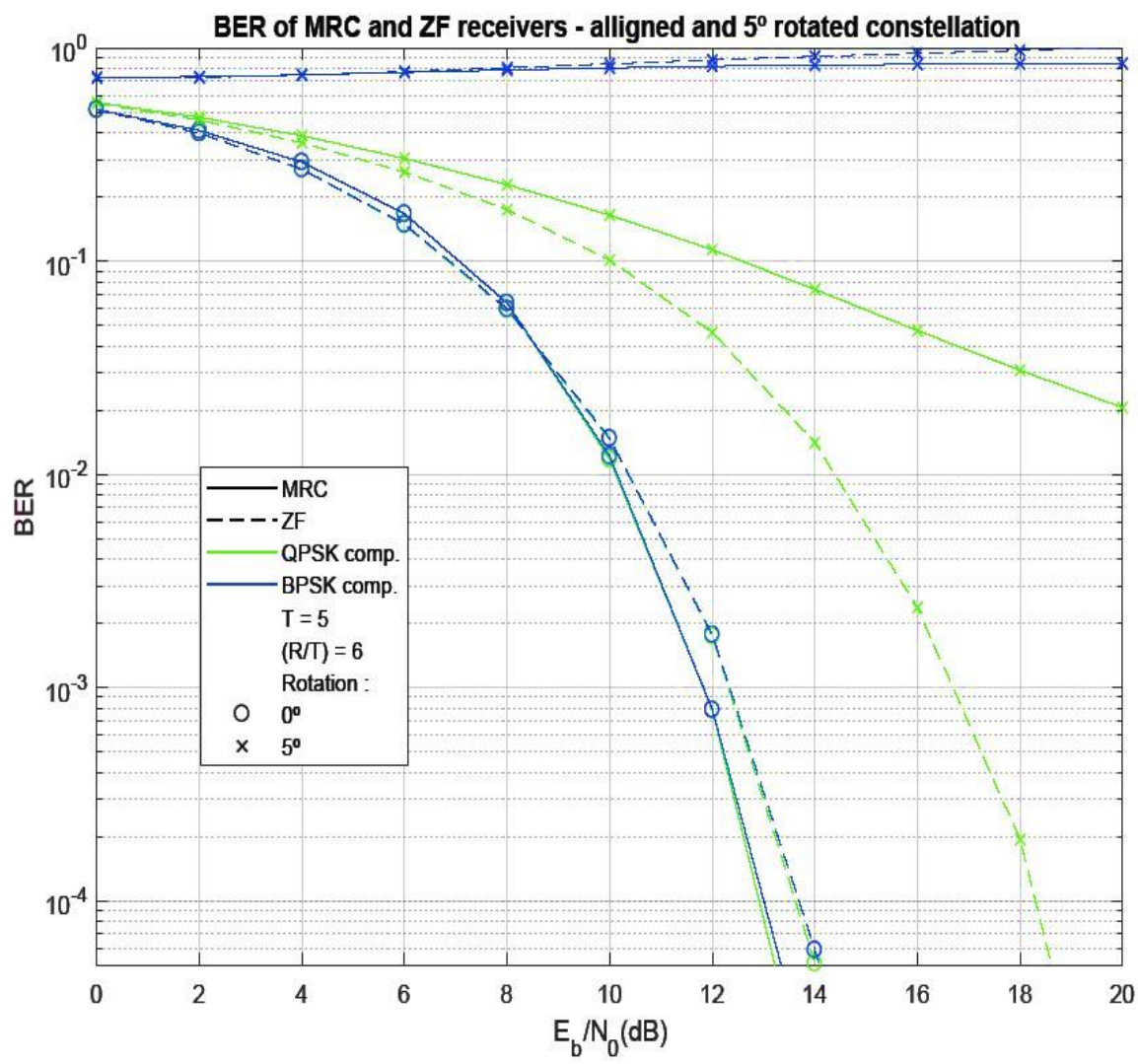

Fig. 8. BER performance for $\mathrm{ZF}$ and MRD with $\Delta \Theta=5^{\circ}$ and variant $R / T$.

Regarding the security level assured by this transmitter, it is evident that the decomposition into BPSK components applied in layer 1 will be more effective than the decomposition into QPSK components. Performance results also show that security due to constellation shaping is achieved independently the type of adopted receiver, since the performance of all receivers is severely affected when $\Delta \Theta \geq 5^{o}$ for $N_{m}=4$. More interestingly, good physical security can be assured in point-to-point communication links with only 4 antennas (for $N_{m}=2$ previous results showed good tolerance against $\Delta \Theta \leq 5^{\circ}$, which can compromise the security). Thus, angular shaping of the transmitted symbol performed in layer 1 can be used to separate user streams and achieve security between them (clearly the angular separation between users should be equal or higher than $5^{o}$ or the the number of components could be increased). Security can be even increased if beamforming is adopted at layer 2 , which will be analyzed in further works.

It should be mentioned that in modern communication systems such as long term evolution (LTE) or further $5 \mathrm{G}$ system, various kinds of channel coding and error 
correction techniques are employed in different layers (mainly in physical and logical layers) to correct residual errors associated to a specific degree of BER. Thus, the BER level of $10^{-4}$ the bit error rate attainable by the proposed tranmission schemes is more than sufficient to achieve the desired quaility of service, since when combined with an error correction we may see much lower error rate than the case without error correction (shown in previous figures). Block error rate (BLER) of frame error rate (FER) are commonly used to assess the system performance, since they result from the sum of contributions of the several error correction techniques employed in the layers. This also means that the previous BER results are within the perfomance ranges employed in physical layer of modern communication systems.

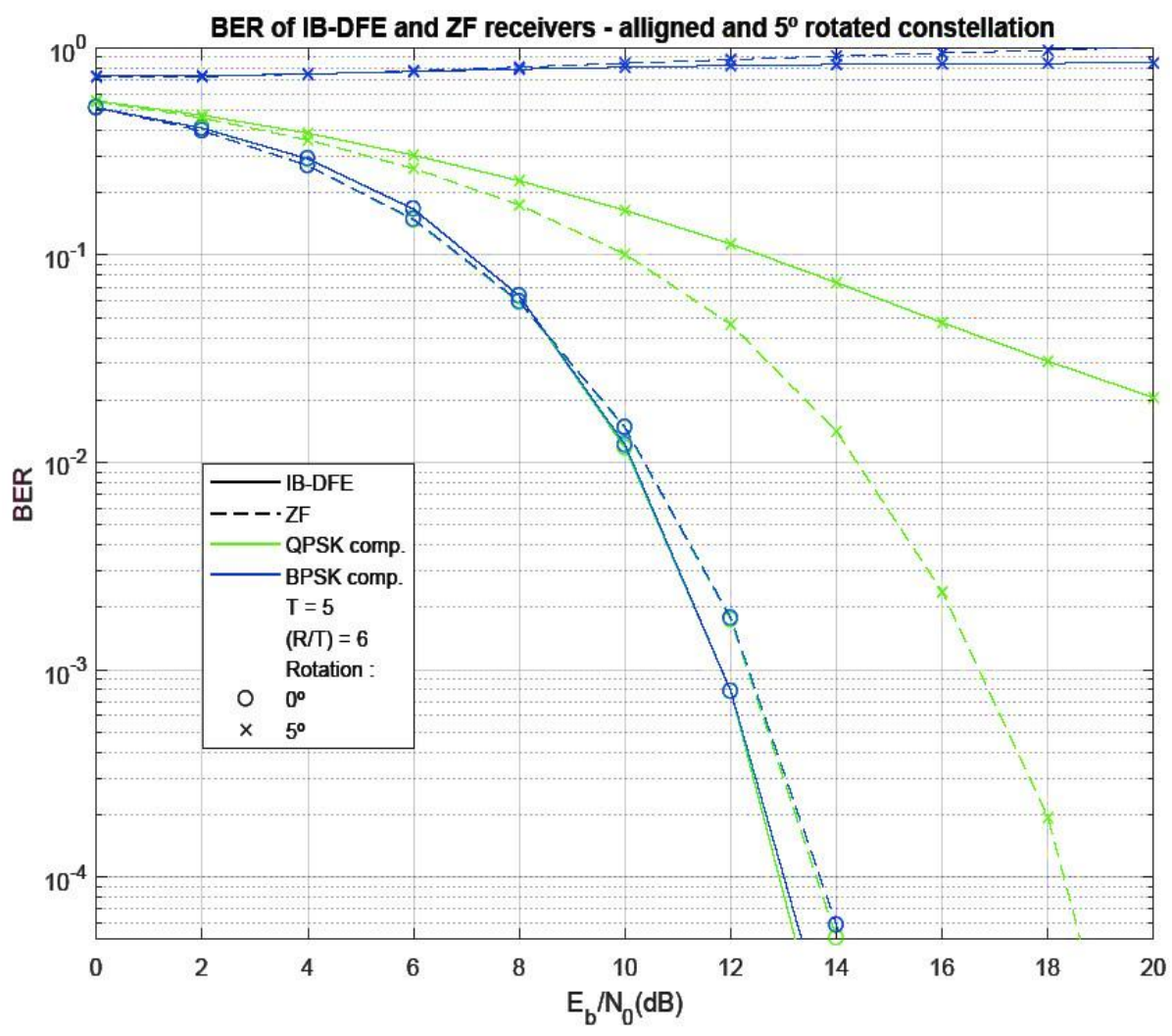

Fig. 9. BER performance for ZF and IB-DFE with $\Delta \Theta=5^{\circ}$ and variant $R / T$. 


\section{Conclusions}

In this paper it was shown that a double layer structure with information directivity and spatial multiplexing could be employed at the transmitter to improve power amplification efficiency and to assure physical security. For an aligned receiver (i.e. $\Delta \Theta=0^{\circ}$ ), since BER results achieved by both decompositions options are similar to those of classical spatial MIMO multiplexing, we may say that both higher power amplification efficiency and physical layer security come without sacrificing system's performance. Despite of using more antennas than the traditional scheme, in further millimetric wave communication systems this factor will be not a restriction.

\section{References}

1. Larsson, Erik G. and Edfors, Ove and Tufvesson, Fredrik and Marzetta, Thomas L., "Massive MIMO for next generation wireless systems", IEEE Communications Magazine, Vol. 52, No. 2, pp.186-195, Feb. 2014.

2. Dinis, R. and Montezuma, P. and Gusmao, A., "Performance trade-offs with quasi-linearly amplified OFDM through a two-branch combining technique", Proc. IEEE VTC96, Atlanta,Georgia, US, May 1996.

3. V. Astucia, P. Montezuma, R. Dinis and M. Beko, "On the use of Multiple grossly Nonlinear amplifiers for Higly Efficient Linear amplification of multilevel constellations", Proc. IEEE VTC2013-Fall, Las Vegas, NV, US, September 2013.

4. R. Dinis, P. Montezuma, N. Souto, and J. Silva,"Iterative Frequency-Domain Equalization for General Constellations", IEEE Sarnoff Symposium, Princeton, USA, Apr. 2010.

5. P. Montezuma and A. Gusmão, "Design of TC-OQAM Schemes Using a Generalised Nonlinear OQPSK-type Format", IEE Elect. Letters, Vol. 35, No. 11, pp. 860-861, May 1999.

6. N. Benvenuto and S. Tomasin, "Block Iterative DFE for Single Carrier Modulation", IEE Elec. Let., Vol. 39, No. 19, pp. 1144-1145, Sep. 2002. 
7. R. Dinis, R. Kalbasi, D. Falconer and A. Banihashemi, "Iterative Layered Space-Time Receivers for Single-Carrier Transmission over Severe Time-Dispersive Channels", IEEE Comm. Letters, Vol. 8, No. 9, pp. 579-581, Sep. 2004.

8. P. Silva, R. Dinis, Frequency-domain multiuser detection for CDMA systems, River Publishers, Aalborg, 2012.

9. F. Silva, R. Dinis and P. Montezuma, "Estimation of the feedback reliability for IB-DFE receivers", ISRN Communications and Networking, Vol. 2011, Article No. 30, Jan. 2011.

10. David Borges, Paulo Montezuma, Rui Dinis, "Low complexity MRC and EGC based receivers for SC-FDE modulations with massive MIMO schemes", 2016 IEEE GlobalSip, Washington, DC, USA, Dec. 2016. 